\title{
Expression of AKT and p-AKT protein in lung adenocarcinoma and its correlation with PD-L1 protein and prognosis
}

\author{
Zhi-Ying Hu ${ }^{1,2}$, Wan-Yi Huang ${ }^{3}$, Lei Zhang ${ }^{4}$, Bo Huang ${ }^{5}$, Shu-Chen Chen ${ }^{1}$, Xiao-Ling Li ${ }^{1}$ \\ ${ }^{1}$ Department of Thoracic Medicine, Cancer Hospital of China Medical University, Liaoning Cancer Hospital and Institute, Shenyang, China; \\ ${ }^{2}$ Department of Respiratory and Critical Care Medicine, Dalian Third People's Hospital, Dalian, China; ${ }^{3}$ Department of aging Science and \\ Pharmacology, Faculty of Dental Science, Kyushu University, Fukuoka, Japan; ${ }^{4}$ Department of Oncology, Shenyang Fifth People Hospital, Shenyang, \\ China; ${ }^{5}$ Department of Pathology, Cancer Hospital of China Medical University, Liaoning Cancer Hospital and Institute, Shenyang, China \\ Contributions: (I) Conception and design: ZY Hu, XL Li; (II) Administrative support: None; (III) Provision of study materials or patients: None; (IV) \\ Collection and assembly of data: None; (V) Data analysis and interpretation: ZY Hu; (VI) Manuscript writing: All authors; (VII) Final approval of \\ manuscript: All authors. \\ Correspondence to: Professor Xiao-Ling Li. Department of Thoracic Medicine, Cancer Hospital of China Medical University, Liaoning Cancer \\ Hospital and Institute, No. 44 Xiaoheyan Road, Dadong District, Shenyang, China. Email: xiaolingli001@163.com.
}

Background: The PI3K/AKT/mTOR signaling pathway were significantly associated with EGFR mutation in lung adenocarcinoma (LUAD), but its correlation with PD-L1 protein and prognosis are not clear. The aim of this study was to evaluate the expression of AKT and phosphorylated AKT (p-AKT) in LUAD and its correlation with programmed death ligand-1 (PD-L1); and to analyze the factors affecting LUAD prognosis.

Methods: The expression of AKT, p-AKT, and PD-L1 was examined using immunohistochemistry in LUAD tissues from 110 patients who underwent surgical treatment.

Results: AKT protein expression was examined in $64.5 \%(71 / 110)$ of the LUAD samples, and p-AKT protein expression was examined in $44.5 \%$ (49/110) of the LUAD samples. The positive rate of PD-L1 at TC1/2/3 was $38.2 \%$ (42/110). AKT and p-AKT expression was significantly associated with epidermal growth factor receptor (EGFR) mutation $(\mathrm{P}=0.016, \mathrm{P}=0.014$ respectively). Pearson's correlation analysis indicated a negative correlation of $\mathrm{p}$-AKT with $\mathrm{PD}-\mathrm{L} 1$ protein $(\mathrm{P}=0.022)$. Out of the 62 patients with EGFR mutation, the expression of PD-L1 was negatively correlated with that of $\mathrm{p}-\mathrm{AKT}$ protein $(\mathrm{P}=0.032)$. The expressions of AKT and p-AKT were not associated with prognosis. Multivariate analysis showed that tumor-node-metastasis $(\mathrm{TNM})$ stage $(\mathrm{P}=0.013)$ and differentiation $(\mathrm{P}=0.046)$ were independent prognostic factors for overall survival.

Conclusions: PI3K/AKT/mTOR in the downstream pathway of EGFR may negatively regulate the expression of PD-L1, which may partly explain why patients with EGFR mutation respond poorly to PD-1/ PD-L1 inhibitors.

Keywords: Lung adenocarcinoma (LUAD); AKT; p-AKT; PD-L1; protein expression; prognosis

Submitted Jul 19, 2020. Accepted for publication Sep 08, 2020.

doi: $10.21037 / \mathrm{atm}-20-5865$

View this article at: http://dx.doi.org/10.21037/atm-20-5865 


\section{Introduction}

Lung adenocarcinoma (LUAD) is the major pathological type of non-small cell lung cancer (NSCLC), with evidence indicating that its global incidence rate is on the rise (1). Despite advances in diagnostic and therapeutic techniques, the 5-year survival rate of LUAD remains unsatisfactory (2). Thus, there is an urgent need for further understanding the molecular mechanisms of LUAD and discovering new molecular targets for treatment. As one of the important signal pathways downstream of epidermal growth factor receptor (EGFR), the PI3K/AKT/mTOR signal pathway has been implicated in a large number of human malignancies including lung cancer, and are involved in tumor angiogenesis, cell proliferation, invasion, and migration. AKT, a serine/threonine protein kinase, is activated in response to activation by many different growth factors, including epidermal growth factor (EGF), fibroblast growth factor (FGF), insulin-like growth factor (IGF), and others. $\mathrm{P}-\mathrm{AKT}$ activates downstream signal factors such as mTOR, p7056k, and 4E-BP1, and transmits survival signals (3).

Therapy based on targeting immune checkpoints, especially anti-programmed death-1 and programmed death ligand-1 (anti-PD-1/PD-L1) monoclonal antibody monotherapy, has made significant progress in the treatment of advanced lung cancer. High expression of PDL1 expression clearly predicts a better chance of benefit from PD-1/PD-L1 inhibitors, although a minority of PDL1-negative patients will still benefit from these agents $(4,5)$. One recent study suggested that patients with EGFR mutations had lower PD-L1 expression (6), while another previous study (7) showed that the presence of EGFR mutant-type is associated with low PD-L1 expression, and the response rate to PD-1/PD-L1 inhibitors is lower than that of wild-type patients. Therefore, it is essential to clarify the PD-L1 expression regulation mechanism in order to obtain more clinical benefits. PI3K/AKT/mTOR pathway, one of the important signal pathways downstream of EGFR, serves as a convergence point for activation of many of the oncogenes involved in NSCLC. Previous studies have suggested that $\mathrm{PI} 3 \mathrm{~K} / \mathrm{AKT} / \mathrm{mTOR}$ pathway may be responsible for regulating PD-L1 expression. NSCLC cell lines with KRAS, EGFR, BRAF, ALK, or RET mutations have been found to express high levels of PD-L1, and this may be linked to a high level of PI3K/AKT/mTOR pathway activation (8). Another previous study showed that activation of the PI3K/AKT/mTOR pathway increased PD-L1 expression in NSCLC (9). A recent study showed that ALK translocation upregulated PD-L1 expression by activating AKT pathways (10). However, in the study by Chen et al. (11), no clear correlation was found between $\mathrm{PD}-\mathrm{L} 1$ protein and $\mathrm{PI} 3 \mathrm{~K} / \mathrm{AKT} / \mathrm{mTOR}$ pathway in NSCLC. The above research results are conflicting, and more studies are needed to further to clarify the relationship between the PI3K/AKT/mTOR pathway and PD-L1. Therefore, in our study, AKT and p-AKT protein were examined by immunohistochemistry, and its correlation with $\mathrm{PD}-\mathrm{L} 1$ protein was investigated to elucidate the underlying correlation.

We present the following article in accordance with the REMARK reporting checklist (available at http://dx.doi. org/10.21037/atm-20-5865).

\section{Methods}

\section{LUAD specimens}

Primary LUAD tissues were collected from 110 patients after curative surgical resection which included mediastinal lymph node dissection at the Liaoning Cancer Hospital and Institute from June 2014 through July 2015. The paraffin tissue blocks of the lung adenocarcinoma patients were stored at room temperature at 4 degrees. The patients were included in the study if the following criteria were fulfilled: (I) the patients with newly diagnosed lung adenocarcinoma cancer who were treated by surgery; (II) the patients who did not receive neoadjuvant therapy before surgery; (III) the patients who underwent EGFR gene mutation test (ARMS test). Exclusion criteria: (I) the patients with incomplete histological and clinical medical records; (II) the patients with neoadjuvant therapy before surgery; (III) the patients with no EGFR gene mutation testing; (IV) the patients with other malignant tumor treatments in the past. Patients included 44 males and 66 females. The average age at diagnosis was 58.6 years (range, 34-78 years). Tumornode-metastasis (TNM) staging revealed stage $\mathrm{I}$ in 58 cases, stage II in 16 cases, and stage III in 36 cases. Lymph node metastasis was observed in 47 cases, while the other 63 cases showed no lymph node metastasis. The tumor diameter was $\leq 3 \mathrm{~cm}$ in 85 cases and $>3 \mathrm{~cm}$ in 25 cases. Poor, moderate, and high differentiation was observed in 28, 44, and 38 cases, respectively. Meanwhile, 62 patients harbored EGFR mutation, while the remaining 48 patients were EGFR wild type. The follow-up period ranged from 5.3 to 56.7 months, with a median time of 46.5 months. At the end of follow-up, 18 patients (16.4\%) were lost to 
Table 1 Clinicopathological characteristics of patients with lung adenocarcinoma

\begin{tabular}{|c|c|c|}
\hline Characteristic & Number & $\%$ \\
\hline \multicolumn{3}{|l|}{ Age } \\
\hline$<60$ & 56 & 50.9 \\
\hline$\geq 60$ & 54 & 49.1 \\
\hline \multicolumn{3}{|l|}{ Gender } \\
\hline Male & 44 & 40.0 \\
\hline Female & 66 & 60.0 \\
\hline \multicolumn{3}{|l|}{ Smoking status } \\
\hline Yes & 37 & 33.6 \\
\hline No & 73 & 66.4 \\
\hline \multicolumn{3}{|l|}{ Stage } \\
\hline I & 58 & 52.7 \\
\hline II & 16 & 14.5 \\
\hline III & 36 & 32.7 \\
\hline \multicolumn{3}{|l|}{ Tumor size $(\mathrm{cm})$} \\
\hline$\leq 3$ & 85 & 77.3 \\
\hline$>3$ & 25 & 22.7 \\
\hline \multicolumn{3}{|l|}{ Tumor differentiation } \\
\hline Poorly differentiated & 28 & 25.5 \\
\hline Moderately differentiated & 44 & 40.0 \\
\hline Well differentiated & 38 & 34.5 \\
\hline \multicolumn{3}{|l|}{ Lymph node metastasis } \\
\hline Yes & 47 & 42.7 \\
\hline No & 63 & 57.3 \\
\hline \multicolumn{3}{|l|}{ EGFR status } \\
\hline Mutant & 62 & 56.4 \\
\hline Wild type & 48 & 43.6 \\
\hline \multicolumn{3}{|l|}{ PD-L1 expression } \\
\hline Positive & 42 & $38.2 \%$ \\
\hline Negative & 68 & $61.8 \%$ \\
\hline
\end{tabular}

EGFR, epidermal growth factor receptor.

follow up and were treated as censored data. This study was approved by the Ethics Committee of Liaoning Cancer Hospital and Institute (Shenyang, China). Informed consent for the experiments were signed by all participants before the study. All procedures performed in this study involving human participants were in accordance with the Declaration of Helsinki (as revised in 2013). The general clinical characteristics of the patients are presented in Table 1.

\section{Immunobistochemistry (IHC)}

IHC staining for AKT, p-AKT, and PD-L1 proteins were performed using the streptavidin-peroxidase method (SP method). Briefly, paraffin-fixed slides were delayed in xylene and dehydrated through graded alcohols. Antigen retrieval was carried out by boiling in a pressure cooker in a citric acid buffer ( $\mathrm{pH} 6.0)$ at $120^{\circ} \mathrm{C}$ for $2 \mathrm{~min}$ and $30 \mathrm{~s}$. Rabbit anti-AKT polyclonal antibody (1:100, AF6261, Affinity Biosciences, USA), rabbit anti-phospho-AKT (Ser473) polyclonal antibody (1:100, AF0908, Affinity Biosciences, USA), and rabbit anti-PD-L1 monoclonal antibody (SP142, Gene Tech Company Limited, China) were incubated on sections for $1 \mathrm{~h}$ at room temperature. Sections were then incubated with the appropriate secondary antibodies at room temperature for $20 \mathrm{~min}$. After sufficient phosphatebuffered saline (PBS) rinses, diaminobenzidine was used as chromogen, and the sections were counterstained with hematoxylin. The slides were counterstained with hematoxylin, and then dehydrated and coverslipped.

\section{Evaluation of immunobistochemical staining}

All slides were blindly interpreted by two independent observers. Prostate cancer sections with high Gleason score and high levels of AKT and p-AKT served as positive controls (12). Sections were immunostained with the omission of the primary antibody as a negative control. The scoring for AKT expression level was determined in the following fashion (13): absence of staining or $<10 \%$ tumor cell staining was scored as negative (-); tumor cell staining of $\geq 10 \%$ and $<50 \%$ was scored as positive (+); and tumor cell staining of $\geq 50 \%$ was scored as strongly positive $(++)$. The scoring for p-AKT (14) was as follows: negative (-), no staining; 1+, weak, homogeneous cytoplasmic staining without a granular staining pattern; $2+$ and $3+$, strong granular cytoplasmic staining with the $2+$ indicating staining in $<20 \%$ of tumor cells and $3+$ indicating staining in $>20 \%$ of tumor cells. We regarded (-) and (1+) as negative expression, while $(2+)$ and $(3+)$ represented positive expression. Placental specimens with PD-L1 expression were used as a positive control. We scored tumor cells expressing PD-L1 as a percentage of total tumor cells. TC1/2/3 was defined as PD-L1 expression on $1 \%$ or more 

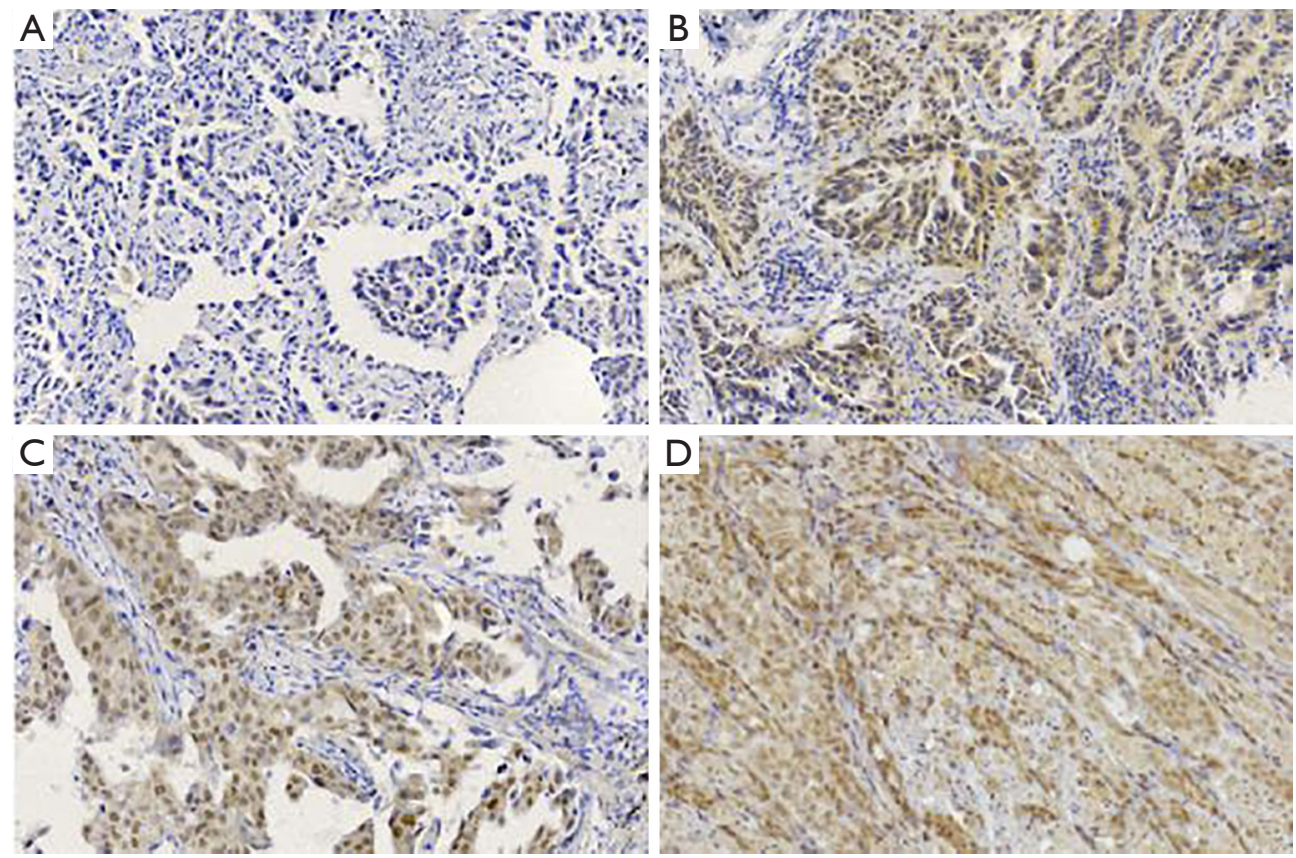

Figure 1 AKT expression in (A) lung adenocarcinoma, (B) lung adenocarcinoma cytoplasm, (C) lung adenocarcinoma nuclei, and (D) prostate cancer. The illustrated sections were interpreted as positive (B, C, and D) or negative (A) for AKT expression. Magnification, $\times 200$.

of tumor cells, TC2/3 was defined as PD-L1 expression in $5 \%$ of tumor cells, TC 3 was defined as PD-L1 expression in $50 \%$ or more of tumor cells, and TC0 was defined as PD$\mathrm{L} 1$ expression in fewer than $1 \%$ of tumor cells. We regarded TC0 as a negative expression, while TC1/2/3 represented positive expression (15).

\section{Statistical analysis}

The experimental data were analyzed by SPSS 20.0 software (IBM, Armonk, NY, USA). The relationships between clinical parameters and expression levels of AKT and p-AKT were analyzed by the $\chi^{2}$ test or Fisher's exact test. The correlation between AKT, p-AKT, and PD-L1 were analyzed by Pearson's correlation analysis. Kaplan-Meier survival curves and the log-rank test were used to analyze overall survival. A cox regression model was utilized to analyze the relationships between the independent risk factors and patient prognosis. Statistical significance was set at $\mathrm{P}<0.05$.

\section{Results}

\section{Expression of AKT, $p-A K T$, and PD-L1}

AKT protein expression was localized mainly in the cytoplasm and was observed in $64.5 \%$ (71/110 cases) of the LUAD samples (Figure 1). The p-AKT protein was expressed in the cytoplasm or nucleus, and was detected in $44.5 \%$ (49/110 cases) of the tumor samples (Figure 2). IHC analysis showed that PD-L1 staining was mainly concentrated in the LUAD cytoplasm and cell membrane (Figure 3). Among the 110 patients, the positive rate of LUAD cells for PD-L1 at TC1/2/3 (positive percentage of tumor cells greater than or equal to $1 \%$ ) was $38.2 \%$ (42/110 cases), PD-L1 in TC2/3 (positive rate of tumor cells greater than or equal to $5 \%)$ was $34.5 \%(38 / 110$ cases), and PD-L1 in TC3 (positive percentage of tumor cells greater than or equal to $50 \%$ ) was $14.5 \%(16 / 110$ cases).

\section{Correlation of AKT and $p-A K T$ expression with protein and clinical pathology characteristics}

In the current research, 62 patients harbored the EGFRsensitive mutation while the remaining 48 cases were EGFR wild type. The expression of AKT and $\mathrm{p}-\mathrm{AKT}$ protein was correlated with EGFR mutation $(\mathrm{P}=0.016, \mathrm{P}=0.014$, respectively), regardless of sex, age, lymph node metastasis, degree of differentiation, or TNM stage (Table 2). 

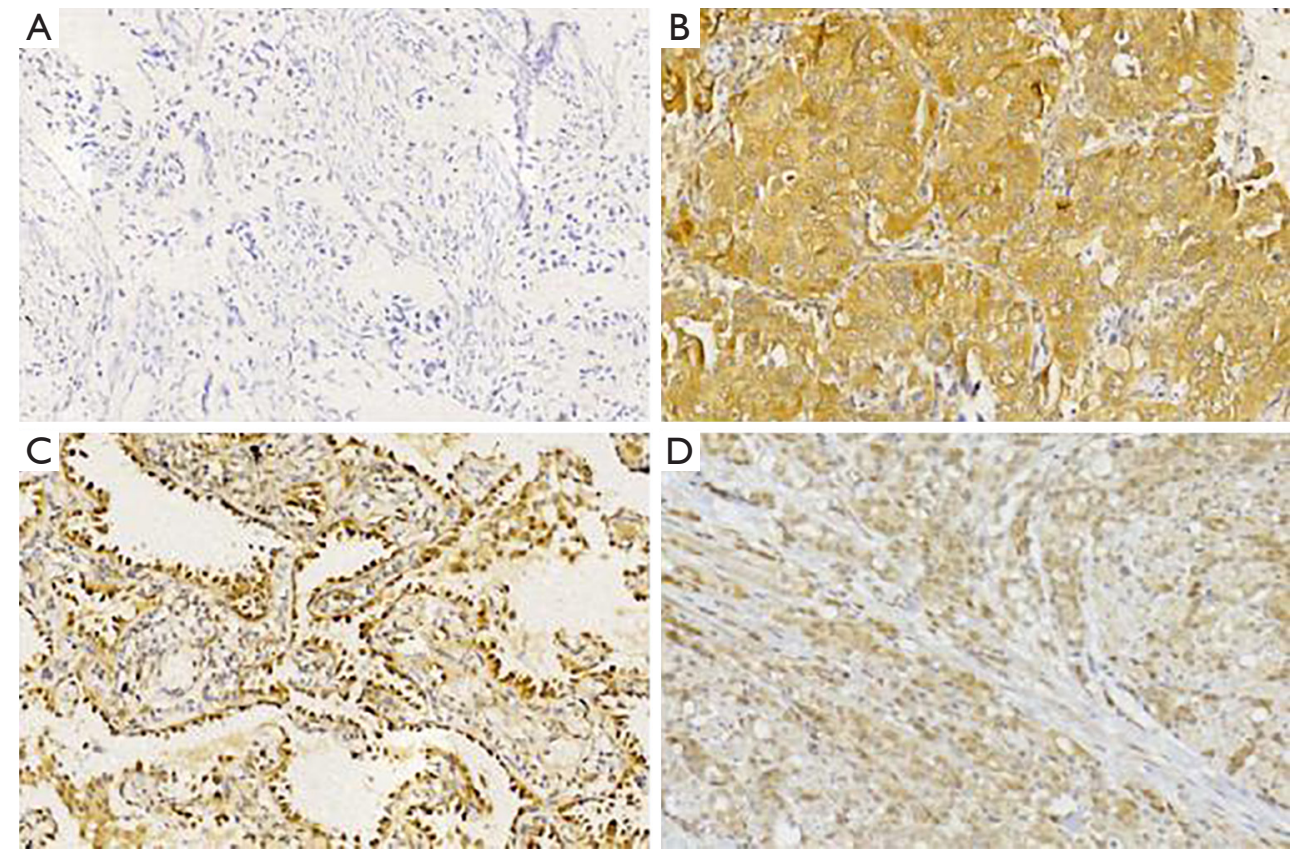

Figure 2 p-AKT expression in (A) lung adenocarcinoma, (B) lung adenocarcinoma cytoplasm, (C) lung adenocarcinoma nuclei, and (D) prostate cancer. The illustrated sections were interpreted as positive (B, C, and D) or negative (A) for p-AKT expression. Magnification, ×200. p-AKT, phosphorylated AKT.
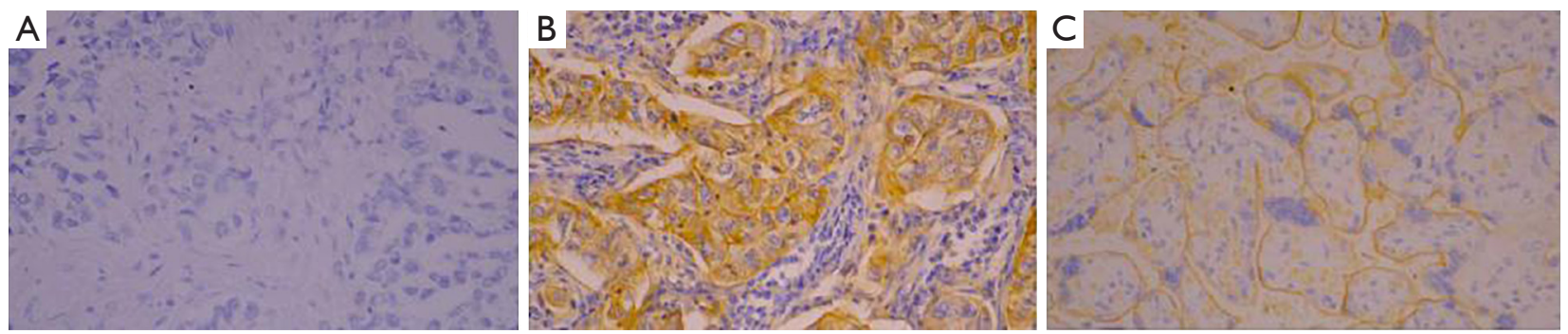

Figure 3 PD-L1 expression in (A) lung adenocarcinoma, (B) lung adenocarcinoma cytoplasm, and (C) placental specimens. The illustrated sections were interpreted as positive (B and C) or negative (A) for PD-L1 expression. Magnification, $\times 400$.

\section{The correlation between AKT, p-AKT, and PD-L1 expression}

Pearson's correlation analysis showed that the expression of AKT was not correlated with that of PD-L1 protein $(\mathrm{r}=-0.025, \mathrm{P}>0.05)$. A negative correlation was found between $\mathrm{p}-\mathrm{AKT}$ and $\mathrm{PD}-\mathrm{L} 1$ protein expression in LUAD ( $\mathrm{r}=-0.128, \mathrm{P}=0.022)$. Out of the 62 patients with EGFR mutation, the expression of PD-L1 was negatively correlated with that of $\mathrm{p}-\mathrm{AKT}$ protein $(\mathrm{r}=-0.272, \mathrm{P}=0.032)$ (Figure 4).

\section{Survival analysis}

For the 110 patients with LUAD, median overall survival was not reached. The 3 -year overall survival rate for the 110 patients was $82.3 \%$. Univariate analysis indicated that TNM stage $(\mathrm{P}=0.000)$, lymph node metastasis $(\mathrm{P}=0.000)$, tumor differentiation $(\mathrm{P}=0.048)$, and tumor diameter $(\mathrm{P}=0.000)$ were significantly associated with poor overall survival (Figure 5). There were no significant differences in AKT $(\mathrm{P}=0.458)$, p-AKT $(\mathrm{P}=0.612)$, gender $(\mathrm{P}=0.794)$, age $(\mathrm{P}=0.123)$, smoking $(\mathrm{P}=0.427)$, and EGFR gene status 
Table 2 Correlation between AKT and p-AKT expression and clinicopathological factors

\begin{tabular}{|c|c|c|c|c|c|c|}
\hline Characteristics & \multicolumn{3}{|c|}{ AKT expression } & \multicolumn{3}{|c|}{$\mathrm{p}-\mathrm{AKT}$ expression } \\
\hline Age & & & 0.392 & & & 0.258 \\
\hline$<60$ & $34(60.7)$ & $22(39.3)$ & & $22(39.3)$ & $34(60.7)$ & \\
\hline$\geq 60$ & $37(68.5)$ & $17(31.5)$ & & $27(50.0)$ & $27(50.0)$ & \\
\hline Male & $26(59.1)$ & $18(40.9)$ & & $19(43.2)$ & $25(56.8)$ & \\
\hline Female & $45(68.2)$ & $21(31.8)$ & & $30(45.5)$ & $36(54.5)$ & \\
\hline Smoking status & & & 0.102 & & & 0.538 \\
\hline Yes & $20(54.1)$ & $17(45.9)$ & & $18(48.6)$ & $19(51.4)$ & \\
\hline I & $35(60.3)$ & $23(39.7)$ & & $31(53.4)$ & 27 (46.6) & \\
\hline II & $9(56.2)$ & $7(43.8)$ & & $4(25.0)$ & $12(75.0)$ & \\
\hline III & $27(75.0)$ & $9(25.0)$ & & $14(38.9)$ & $22(61.1)$ & \\
\hline Tumor size $(\mathrm{cm})$ & & & 0.589 & & & 0.151 \\
\hline$\leq 3$ & $56(65.9)$ & $29(34.1)$ & & $41(48.2)$ & $44(51.8)$ & \\
\hline$>3$ & $15(60.0)$ & $10(40.0)$ & & $8(32.0)$ & $17(68.0)$ & \\
\hline Degree of differentiation & & & 0.151 & & & 0.976 \\
\hline Low & $19(67.9)$ & $9(32.1)$ & & $12(42.9)$ & $16(57.1)$ & \\
\hline No & $38(60.3)$ & $25(39.7)$ & & $33(52.4)$ & $30(47.6)$ & \\
\hline EGFR status & & & $0.016^{*}$ & & & $0.014^{*}$ \\
\hline Mutant & $46(74.2)$ & $16(25.8)$ & & $34(54.8)$ & $28(45.2)$ & \\
\hline Wild type & $25(52.1)$ & $23(47.9)$ & & 15 (31.3) & $33(68.7)$ & \\
\hline
\end{tabular}

*, P<0.05. p-AKT, phosphorylated AKT; EGFR, epidermal growth factor receptor.

$(\mathrm{P}=0.470)$. By multivariate analysis, TNM stage $(\mathrm{P}=0.013)$ and degree of differentiation $(\mathrm{P}=0.046)$ were revealed to be independent prognostic factors for overall survival.

\section{Discussion}

The investigation of molecular mechanisms underlying lung cancer development and progression is vital. A growing body of evidence indicates that AKT is a cytosolic signal transduction protein that figures prominently in mechanisms of carcinogenesis and chemoresistance. The present study revealed that the positive staining of AKT and p-AKT was observed predominantly in the cytoplasm and occasionally in the nucleus. AKT is overexpressed in various types of human cancer such as lung cancer, breast cancer, and prostate cancer $(12,13,16,17)$. Previous studies have reported positive rates of AKT between $52.1 \%$ and $93.3 \%$ $(13,18,19)$ and positive rates of p-AKT between $33.3 \%$ and 

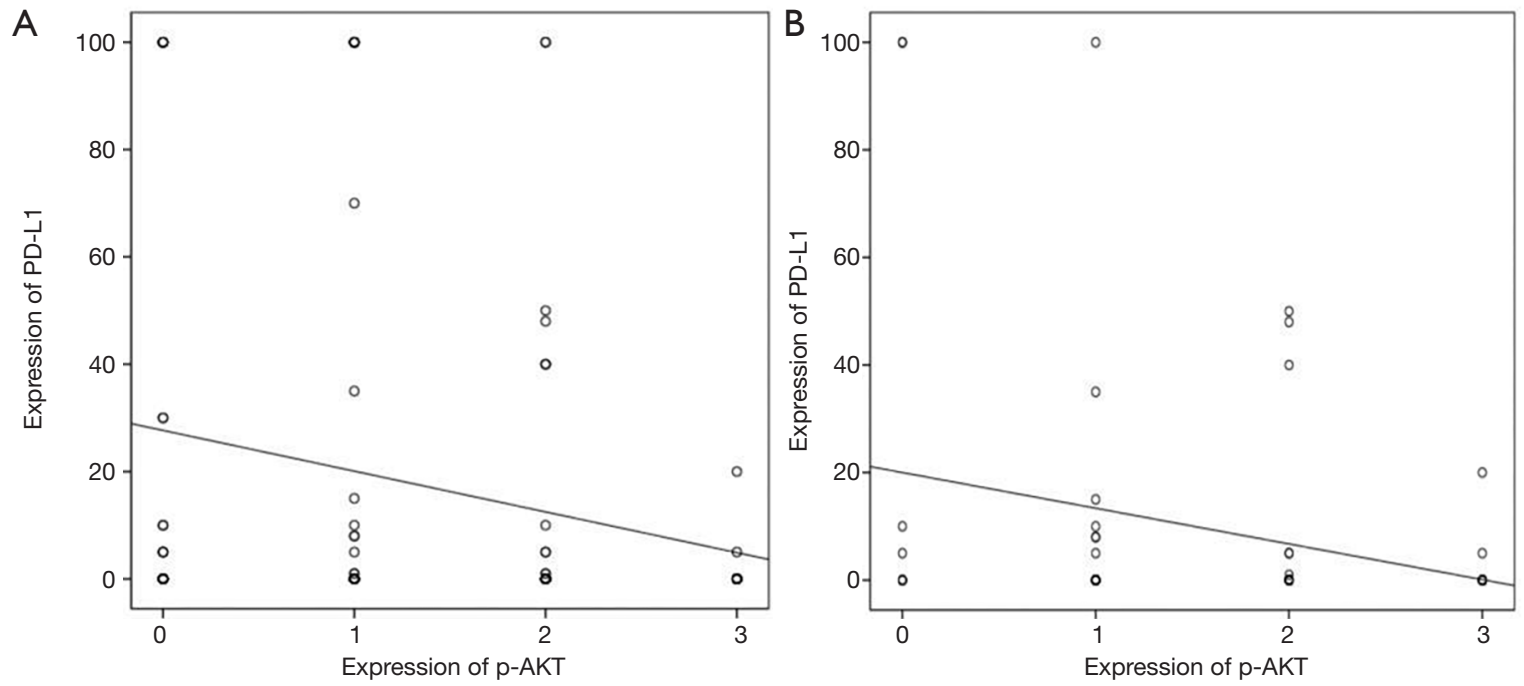

Figure 4 Correlation of PD-L1 and p-AKT expression in: lung adenocarcinoma (A), lung adenocarcinoma with the EGFR mutation (B). p-AKT, phosphorylated AKT; EGFR, epidermal growth factor receptor.
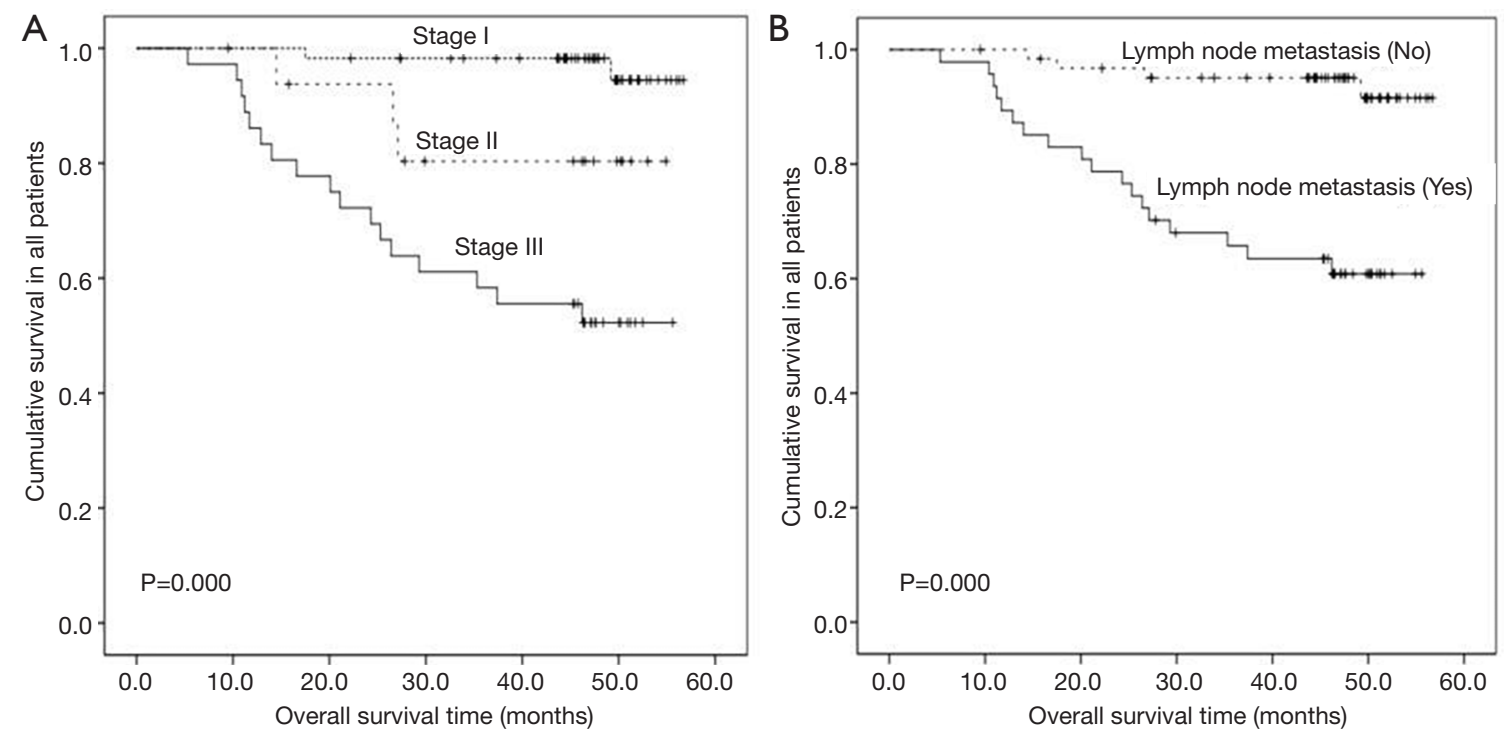

Figure 5 Kaplan-Meier survival curves for overall survival rate and the results of the log-rank test.

$87.5 \%(13,20-22)$. Our data revealed that AKT and p-AKT were highly expressed in LUAD tissues, which is consistent with previous reports. Moreover, AKT and p-AKT were significantly associated with EGFR mutation, which is in line with the results of Sordella et al. (23) and Zhao et al. (16). This indicates that EGFR mutation can activate the downstream PI3K/AKT/mTOR signaling pathway, and lead to the overexpression of AKT and p-AKT, thereby promoting the development of lung cancer. Previous studies
$(16,21,22)$ have found that the expression of p-AKT in NSCLC is closely related to poor differentiation and lymph node metastasis, and it is believed that the activation of AKT plays an important role in the development of lung cancer. Other studies, however, have observed the opposite effect. Mukohara et al. (24) found that the expression of p-AKT was associated with high differentiation and early stage, but not lymph node metastasis. In addition, Tsao et al. (25) showed that p-AKT was highly expressed in 
NSCLC tissues and premalignant bronchial epithelial tissues, but was barely expressed in normal cells, suggesting that AKT activation is involved in the precancerous lesion process of bronchial epithelial cells, which precipitates the occurrence of NSCLC. In the present study, no correlation between AKT and p-AKT expression and stage, degree of differentiation, and lymph node metastasis was found. The variability in pathological types and clinicopathological features studied may be the cause of these inconsistent conclusions.

There is still controversy concerning the significance of AKT-positive expression for the prognosis of NSCLC. Most studies suggest that the overactivation of AKT predicts a poor prognosis for NSCLC $(20,21,26,27)$, but Shah et al. (28) and Cappuzzo et al. (29) believe that patients with high p-AKT expression have good prognosis. In addition, some studies have concluded that p-AKTpositive expression is not associated with the prognosis of NSCLC $(14,30,31)$. Our results suggested that the positive expression of AKT and p-AKT protein in LUAD was not associated with prognosis. Many factors can contribute to conflicting research results, such as a heterogeneity in the clinical and pathological features of the study subjects, the number of selected samples, experimental methods, experimental reagents, and interpretation criteria.

Research into PD-1/PD-L1 pathway is currently the fastest growing and most mature of the studies into immunological checkpoint pathways being conducted, and this research has figured prominently in tumor immunotherapy. The importance of PD-L1 is reflected by the antitumor activity observed when PD-L1 blocking antibodies are used. This cell surface protein is upregulated in many epithelial tumors such as breast cancer (32), colon cancer (33), and NSCLC (34). Our study showed that the positive rate of PD-L1 expression in LUAD cells was $38.2 \%$, which was consistent with a previous study (35). However, the regulation mechanism of PD-L1 expression is unclear, the multiple mechanisms may be involved which includes the status of underlying transcriptional and signaling networks. The published study showed that EGFR mutation was correlated with lack of T-cell infifiltration, decreased PD-L1 expression and lower TMB, which were predictive biomarkers for PD-1 blockade immunotherapy (6), however, the molecular mechanism of PD-L1 mediated by EGFR in NSCLC was not illustrated in this paper. Another report showed that PDL1 expression was regulated by ERK, AKT, and STAT3 signaling pathways in NSCLC with ALK translocation (10).
Our study found that LUAD with EGFR mutation had high expression of p-AKT and low level of PD-L1 protein, and the expression of p-AKT protein negatively correlated with PD-L1. This finding differs from that of previous study, which found that PI $3 \mathrm{~K} / \mathrm{AKT} / \mathrm{mTOR}$ increases the expression of PD-L1 (8). This difference is likely due to the heterogeneity of the study populations. According to the results of our study, we speculated that mutated EGFR activated the downstream PI3K/AKT/mTOR signal pathway, upregulated the expression of $\mathrm{p}$-AKT protein and then negatively regulated the expression of PD-L1, which to some extent explains why NSCLC patients with positive EGFR-sensitive mutation respond poorly to $\mathrm{PD}-1 /$ PD-L1 inhibitors. Conversely, we speculate that PD-L1 expression may be upregulated after EGFR-TKI treatment, and post-TKI treatment patients may benefit from PD-1/ PD-L1 inhibitors. This potential for clinical benefit supports efforts to study the mechanisms that regulate PDL1 expression and therapeutic interventions to increase PD-L1 levels. The components of PI3K/AKT/mTOR signaling pathway are complex, and there are many cascadeeffect proteins in the pathway, which are involved in the physiological and pathological process of cells. In this study, only the correlation between AKT, p-AKT, and PD-L1 was analyzed, while the correlations between other signal transduction proteins and PD-L1 are not yet clear, and should be further studied.

Our study has several limitations. The subjects of this cohort were patients with stage I-III LUAD after radical operation, with stage I-II patients accounting for a relatively high proportion; there was also a lack of stage IV patients. The sample size of this study was relatively small, consequently, these results required further validation in a large cohort. But it showed a trend that LUAD patients with EGFR mutation had lower PD-L1 expression, which was consistent with previous studies (6,7). In addition, we only analyzed the relationship between AKT, p-AKT, and PD-L1 in the archive wax block at the protein level. In the follow-up, we can explore the correlation of p-AKT and PD-L1 in LUAD at the cellular level and gene level to clarify the underlying regulatory mechanism.

In conclusion, the present study demonstrated that AKT and p-AKT were overexpressed and positively correlated with EGFR mutation in LUAD tissues. A negative correlation was established between p-AKT and PD-L1 protein, which was also found in LUAD cases with the EGFR mutation. These data suggest that EGFR mutation activates the downstream PI3K/AKT/mTOR signaling 
pathway, and then decreases the expression of PD-L1. This partly explains why patients with EGFR mutation respond poorly to PD-1/PD-L1 inhibitors. Post-EGFR-TKItreatment patients may thus benefit from PD-1/PD-L1 inhibitors, as their PD-L1 expression may be upregulated. Despite these promising findings, a large number of clinical studies are still required to further verify our conclusions.

\section{Acknowledgments}

The authors gratefully acknowledge the time and energy contributed by the participants.

Funding: None.

\section{Footnote}

Reporting Checklist: The authors have completed the REMARK reporting checklist. Available at http://dx.doi. org/10.21037/atm-20-5865

Data Sharing Statement: Available at http://dx.doi. org/10.21037/atm-20-5865

Conflicts of Interest: All authors have completed the ICMJE uniform disclosure form (available at http://dx.doi. org/10.21037/atm-20-5865). The authors have no conflicts of interest to declare.

Ethical Statement: The authors are accountable for all aspects of the work in ensuring that questions related to the accuracy or integrity of any part of the work are appropriately investigated and resolved. The present study was approved by the Ethics Review Committee of the Cancer Hospital of China Medical University (Shenyang, China). Written informed consent was obtained from all patients included within the present study. All procedures performed in this study involving human participants were in accordance with the Declaration of Helsinki (as revised in 2013).

Open Access Statement: This is an Open Access article distributed in accordance with the Creative Commons Attribution-NonCommercial-NoDerivs 4.0 International License (CC BY-NC-ND 4.0), which permits the noncommercial replication and distribution of the article with the strict proviso that no changes or edits are made and the original work is properly cited (including links to both the formal publication through the relevant DOI and the license). See: https://creativecommons.org/licenses/by-nc$\mathrm{nd} / 4.0 /$.

\section{References}

1. Johnson DH, Schiller JH, Bunn PA Jr. Recent clinical advances in lung cancer management. J Clin Oncol 2014;32:973-82.

2. Ma L, Chen R, Wang F, et al. EGFR L718Q mutation occurs without T790M mutation in a lung adenocarcinoma patient with acquired resistance to osimertinib. Ann Transl Med 2019;7:207.

3. Andjelković M, Alessi DR, Meier R, et al. Role of translocation in the activation and function of protein kinase B. J Biol Chem 1997;272:31515-24.

4. Areses Manrique MC, Mosquera Martínez J, García González J, et al. Real world data of nivolumab for previously treated non-small cell lung cancer patients: a Galician lung cancer group clinical experience. Transl Lung Cancer Res 2018;7:404-15.

5. Garon EB, Rizvi NA, Hui R, et al. Pembrolizumab for the Treatment of Non-Small-Cell Lung Cancer. N Engl J Med 2015;372:2018-28.

6. Dong ZY, Zhang JT, Liu SY, et al. EGFR mutation correlates with uninflamed phenotype and weak immunogenicity, causing impaired response to PD-1 blockade in non-small cell lung cancer. Oncoimmunology 2017;6:e1356145.

7. Jin $Y$, Shen X, Pan Y, et al. Correlation between PDL1 expression and clinicopathological characteristics of non-small cell lung cancer: A real-world study of a large Chinese cohort. J Thorac Dis 2019;11:4591-601.

8. Brahmer JR, Tykodi SS, Chow LQM, et al. Safety and Activity of Anti-PD-L1 Antibody in Patients with Advanced Cancer. N Engl J Med 2012;366:2455-65.

9. Lastwika KJ, Wilson W, Li QK, et al. Control of PDL1 expression by oncogenic activation of the AKT/ mTOR pathway in non-small cell lung cancer. Cancer Res 2016;76:227-38.

10. Ma L, Lv J,Dong Y, et al. PD-L1 Expression and Its Regulation in Lung Adenocarcinoma with ALK Translocation. Interdiscip Sci 2019;11:266-72.

11. Chen N, Fang W, Zhan J, et al. Upregulation of PDL1 by EGFR Activation Mediates the Immune Escape in EGFR-Driven NSCLC: Implication for Optional Immune Targeted Therapy for NSCLC Patients with EGFR Mutation. J Thorac Oncol 2015;10:910-23.

12. Malik SN, Brattain M, Ghosh PM, et al. 
Immunohistochemical demonstration of phospho-Akt in high Gleason grade prostate cancer. Clin Cancer Res 2002;8:1168-71.

13. Dobashi Y, Tsubochi H, Matsubara H, et al. Diverse involvement of isoforms and gene aberrations of Akt in human lung carcinomas. Cancer Sci 2015;106:772-81.

14. Hirami Y, Aoe M, Tsukuda K, et al. Relation of epidermal growth factor receptor, phosphorylated-Akt, and hypoxiainducible factor-1alpha in non-small cell lung cancers. Cancer Lett 2004;214:157-64.

15. Herbst RS, Soria JC, Kowanetz M, et al. Predictive correlates of response to the anti-PD-L1 antibody MPDL3280A in cancer patients. Nature 2014;515:563-7.

16. Zhao XD, Deng HB, Lu CL, et al. Association of EGFR and KRAS mutations with expression of p-AKT, DR5 and DcR1 in non-small cell lung cancer. Neoplasma 2017;64:182-91.

17. Pérez-Tenorio G, Stål O, et al. Southeast Sweden Breast Cancer Group. Activation of AKT/PKB in breast cancer predicts a worse outcome among endocrine treated patients. Br J Cancer 2002;86:540-5.

18. Dobashi Y, Kimura M, Matsubara H, et al. Molecular alterations in AKT and its protein activation in human lung carcinomas. Hum Pathol 2012;43:2229-40.

19. Yu X, Yuan Y, Zhi X, et al. Correlation between the protein expression of A-kinase anchor protein 95, cyclin D3 and AKT and pathological indicators in lung cancer tissues. Exp Ther Med 2015;10:1175.

20. Yoshizawa A, Fukuoka J, Shimizu S, et al. Overexpression of phospho-eIF4E is associated with survival through AKT pathway in non-small cell lung cancer. Clin Cancer Res 2010;16:240-8.

21. Tang JM, He QY, Guo RX, et al. Phosphorylated Akt overexpression and loss of PTEN expression in non-small cell lung cancer confers poor prognosis. Lung Cancer 2006;51:181-91.

22. Yun F, Jia Y, Li X, et al. Clinicopathological significance of PTEN and PI3K/AKT signal transduction pathway in non-small cell lung cancer. Int J Clin Exp Pathol 2013;6:2112-20.

23. Sordella R, Bell DW, et al. Haber DA Gefitinib-Sensitizing EGFR Mutations in Lung Cancer Activate Anti-Apoptotic Pathways. Science 2004;305:1163-7.

24. Mukohara T, Kudoh S, Matsuura K, et al. Activated Akt expression has significant correlation with EGFR and TGF-alpha expressions in stage I NSCLC. Anticancer Res 2004;24:11-7.

25. Tsao AS, McDonnell T, Lam S, et al. Increased phospho-
AKT (Ser(473)) expression in bronchial dysplasia: implications for lung cancer prevention studies. Cancer Epidemiol Biomarkers Prev 2003;12:660.

26. Liu K, Chen HL, Gu MM, et al. A three gene-based risk score predicts prognosis of resected non- small-cell lung cancer. Int J Clin Exp Pathol 2015;8:16081.

27. Tsurutani J, Fukuoka J, Tsurutani H, et al. Evaluation of two phosphorylation sites improves the prognostic significance of Akt activation in non-small-cell lung cancer tumors. J Clin Oncol 2006;24:306.

28. Shah A, Swain WA, Richardson D, et al. Phospho-Akt expression is associated with a favorable outcome in nonsmall cell lung cancer. Clin Cancer Res 2005;11:2930-6.

29. Cappuzzo F, Magrini E, Ceresoli GL, et al. Akt Phosphorylation and Gefitinib Efficacy in Patients With Advanced Non-Small-Cell Lung Cancer. J Natl Cancer Inst 2004;96:1133-41.

30. Hosokawa S, Toyooka S, Fujiwara Y, et al. Comprehensive analysis of EGFR signaling pathways in Japanese patients with non-small cell lung cancer. Lung Cancer 2009;66:107-13.

31. Kitano H, Chung JY, Ylaya K, et al. Profiling of phosphoAKT, phospho-mTOR, phospho-MAPK and EGFR in non-small cell lung cancer. J Histochem Cytochem 2014;62:335-46.

32. Zhang M, Sun H, Zhao S, et al. Expression of PD-L1 and prognosis in breast cancer: a meta-analysis. Oncotarget 2017;8:31347-54.

33. Wang L, Ren F, Wang Q, et al. Significance of Programmed Death Ligand 1 (PD-L1) Immunohistochemical Expression in Colorectal Cancer. Mol Diagn Ther 2016;20:175-81.

34. Lafuente-Sanchis A, Zúñiga Á, Estors M, et al. Association of PD-1, PD-L1, and CTLA-4 Gene Expression and Clinicopathologic Characteristics in Patients With Non-Small-Cell Lung Cancer. Clin Lung Cancer 2017;18:e109.

35. Fehrenbacher L, Spira A, Ballinger M, et al. Atezolizumab versus docetaxel for patients with previously treated non-small-cell lung cancer (POPLAR): a multicentre, open-label, phase 2 randomised controlled trial. Lancet 2016;387:1837-46.

Cite this article as: $\mathrm{Hu} \mathrm{ZY}$, Huang WY, Zhang L, Huang B, Chen SC, Li XL. Expression of AKT and p-AKT protein in lung adenocarcinoma and its correlation with PD-L1 protein and prognosis. Ann Transl Med 2020;8(18):1172. doi: 10.21037/ atm-20-5865 